\title{
Imagination et lecture selon Ingarden : La délicatesse de l'imagination
}

Par ANNE COIGNARD

Université de Toulouse - Jean Jaurès, EA ERRaPhiS

Résumé Nous proposons dans cet article d'aborder le traitement que Roman Ingarden réserve à l'imagination dans ses travaux sur l'œuvre littéraire et l'expérience de lecture littéraire. Il s'agit, d'abord, de revenir sur la conception qu'il propose de la stratification de l'œuvre littéraire, pour examiner la manière dont chaque strate en appelle à des actes d'imagination spécifiques et pour tenter de décrire l'entrelacement de ces divers actes au cours de la lecture. Mais parce qu'Ingarden ne thématise pas seulement la pluralité des mises en œuvre de l'imagination, mais aussi la pluralité des attitudes de lecteur, nous questionnons, dans un second temps, la manière dont il hiérarchise les lecteurs et les manières de lire en établissant une distinction stricte entre usage esthétique et usage non-esthétique de l'imagination. Afin de faire ressortir le fait que cette distinction même témoigne, chez Ingarden, de l'insistance de présupposés issus de l'esthétique classique, nous proposons, dans une dernière étape, de le confronter à Hume, afin de montrer comment il en appelle, dans son approche de l'expérience littéraire, à une certaine forme de délicatesse dans le travail de l'imagination.

\section{Introduction}

Au premier abord, il pourrait sembler que l'imagination de celui qui lit une œuvre d'art littéraire soit sollicitée lorsqu'il se figure ce qu'il lit. Le lecteur de roman, par exemple, mettrait en mouvement son imagination pour se figurer les personnages, les lieux de l'intrigue, les scènes qui suscitent son intérêt, etc. L'imagination lectrice se cantonnerait ainsi à la production d'un remplissement intuitif pour le texte qui est tenu entre les mains. 
Or, à prendre en compte les travaux de Roman Ingarden sur l'œuvre d'art littéraire, qui insistent sur la stratification de celle-ci et sur la multiplicité d'actes qu'elle requiert de la part du lecteur, nous sommes amenée à concevoir des usages distincts et entrelacés de l'imagination dont la fécondité, lorsqu'elle est motivée par la lecture d'un texte littéraire, ne se réduit pas à produire les images qui flottent dans la tête du lecteur. L'approche d'Ingarden nous invite à concevoir une pluralité d'actes d'imagination dont les modalités doivent être à chaque fois décrites, de telle sorte qu'il s'agit d'articuler ontologie de l'œuvre littéraire et phénoménologie de la lecture.

À examiner de près l'approche d'Ingarden, on aperçoit cependant que ses élaborations ontologiques et phénoménologiques sont entièrement soustendues par des enjeux normatifs. L'enjeu, pour lui, n'est jamais seulement de dire en quoi consiste une œuvre littéraire ou de décrire l'expérience de lecture dans la pluralité de ses dimensions. L'œuvre est l'autre nom d'une exigence, de telle sorte que la phénoménologie de la lecture qui se déploie est animée par le souci de décrire ce qui se passe quand l'expérience de lecture rend justice à l'œuvre lue, voire de décrire ce qui devrait se passer pour qu'une juste lecture ait lieu. C'est ainsi qu'il ne s'agit pas seulement, pour Ingarden, de dire comment l'imagination du lecteur joue pendant la lecture. Ingarden met en scène des figures de lecteur - figures de bons et de mauvais lecteurs - qui permettent de distinguer entre des usages légitimes et illégitimes de l'imagination. En cela, l'approche d'Ingarden marque sa proximité avec l'esthétique classique, en ce qu'elle s'efforce d'énoncer les critères d'un bon usage de l'imagination dans la lecture.

Toute la question est alors de savoir dans quelle mesure cette approche est tenable. Peut-on sans plus de difficultés articuler une phénoménologie de la lecture à une réflexion sur les normes de la bonne lecture ? Peut-on choisir, en tant que phénoménologue, de privilégier certains lecteurs plutôt que d'autres, voire peut-on élire certains lecteurs comme sujet légitimes de la description phénoménologique ? Ce sont ces questions qui nous guideront ici, au cours d'un développement qui visera à faire ressortir les tensions qui traversent l'approche d'Ingarden et à manifester la difficulté qu'il y a, lorsqu'on aborde la lecture littéraire, à ne pas faire intervenir immédiatement la question de l'évaluation des œuvres et des lectures.

Nous proposons, dans un premier moment, de revenir sur la stratification de l'œuvre d'art littéraire telle qu'elle est exposée par Ingarden afin de mettre en lumière la manière dont l'imagination du lecteur est sollicitée par chacune de ses strates. Dans un second moment, nous nous arrêterons sur la notion de concrétisation, afin d'examiner la manière dont Ingarden ne distingue pas seulement entre les divers usages de l'imagination, tels qu'ils 
sont requis pas les diverses strates, mais opère un partage entre usages légitimes et illégitimes de l'imagination selon les lecteurs. Cela nous conduira, dans un dernier moment, à opérer une rapide mise en dialogue d'Ingarden et Hume, afin de peser ce que l'approche d'Ingarden doit encore, dans ses présupposés même, à l'esthétique classique.

\section{Strates de l'œuvre littéraire et entrelacement des actes d'imagination}

L'œuvre littéraire, telle qu'elle est conçue par Ingarden, est constituée de diverses strates, de telle sorte qu'elle en appelle à une constitution elle-même composée de divers actes d'appréhension fondés les uns sur les autres et entrelacés. Ingarden identifie en effet, dans L'Euvre d'art littéraire, quatre strates : la strate des vocables (Worlaute) en tant qu'elle tisse un matériau phonique, celle des unités de signification (Wortbedeutungen), celle des objectivités figurées (dargestellte Gegenständlichkeiten) par les significations du texte, soit la strate des corrélats objectifs des phrases, et enfin celle des aspects (Ansichten) sous lesquels ces objets apparaissent ${ }^{1}$.

Dans les quelques paragraphes qui suivent, afin d'aborder l'imagination telle qu'elle est mise en mouvement par la lecture d'une œuvre littéraire, il s'agit de questionner chaque strate afin de déterminer dans quelle mesure et comment elle sollicite l'imagination du lecteur.

\subsection{Lire : écouter en imagination}

Ingarden, pour dire la première strate, parle de vocables afin de mettre en avant la nécessité de prendre en compte la qualité sensible des mots dont le texte est fait. En littérature - mais en est-il vraiment autrement ailleurs ? les mots ne sont pas simplement là pour signifier mais, aussi et toujours, pour être entendus. C'est à cela que l'on décèle une première manière pour l'imagination du lecteur d'être sollicitée par l'œuvre littéraire. Cette dernière, si elle n'est pas toujours proférée à haute voix, se doit d'être entendue dans ses harmonies sonores, déchiffrée et accompagné dans son rythme; elle se doit d'être saisie dans cette voix intérieure qui accompagne toute lecture et fait résonner les mots. Comme l'écrit Ingarden, la strate des vocables «n'est pas

${ }^{1}$ R. Ingarden, L'CEuvre d'art littéraire, tr. fr. P. Secretan avec la collaboration de N. Lüchinger et B. Schwegler, Lausanne, L'Âge d'Homme, 1983, p. 44. 
seulement un moyen de dévoilement de l'œuvre littéraire ${ }^{1}$, un médium voué à s'effacer devant la saisie des significations. La strate des vocables, parce qu'elle est le lieu de ce qu'il nomme des phénomènes glossophoniques - rythmes, tempo, qualités affectives - , ne consiste nullement en une strate qui devrait être dépassée, et surtout oubliée, dans la compréhension ou la visée du sens ${ }^{2}$. Les vocables, pour autant qu'on leur prête attention en tant qu'objets sonores, ou qu'on préserve à même la lecture leur dimension sonore, constituent cette chair du texte, cette présence sensible de l'œuvre dont la lecture est aussi l'expérience.

Un texte n'est jamais lu sans être entendu et l'imagination lectrice intervient, à même cette strate, pour faire entendre la voix intérieure de la lecture. En premier ressort donc, et avant toute figuration d'objet, l'imagination est projective d'une voix par laquelle le texte prend chair dans l'intériorité même du lecteur. Le premier acte d'imagination du lecteur consiste donc à se donner à entendre le texte et, plus que cela, à trouver le rythme et la voix qui conviennent à sa lecture. Comme un musicien qui accorde son instrument pour le morceau qu'il s'apprête à jouer, le lecteur doit, en tâtonnant, à même une écoute intérieure, trouver la voix du texte, la manière de le faire résonner et de le rendre présent.

L'imagination telle qu'elle est mise en mouvement par la première strate de l'œuvre littéraire permet donc au lecteur d'entrer dans l'œuvre, d'abord, comme dans un monde sonore. Et il s'agit bien là de l'entrée dans un autre monde - avant même qu'une imagination productrice d'images fasse flotter dans la tête du lecteur des objets qu'il ne saurait actuellement percevoir. En effet, c'est bien parce que se joue, au fur et à mesure de l'immersion dans la lecture comme récitation intérieure, une animation rythmique et harmonique du lecteur, que la lecture provoque la rupture avec l'expérience actuelle perceptive et avec les préoccupations du jour. Nous n'entrons pas seulement dans la lecture parce que ce dont parle le texte nous intéresse, parce que ce qu'il nous donne à présentifier capte notre attention. Avant tout chose, c'est parce que, dans le silence même de la lecture, nous entendons une voix qui impose sa présence contre les bruits du monde environnant, que nous sommes emportés ailleurs, littéralement séduits ou charmés. La concentration du lecteur, son absorption en apparence silencieuse manifestent sa captation par le flux sonore que son imagination, à mesure qu'il lit les phrases du texte, déploie dans l'espace intérieur de sa lecture.

${ }^{1}$ Ibid., § 14, p. 65.

${ }^{2}$ Ibid., § 12, p. 62. 


\subsection{Faire résonner les mots : affectivité et compréhension}

Les vocables ne sont cependant pas constitués d'une seule face sonore. S'il est impossible de lire sans entendre les mots, de telle sorte que le lecteur écoute sa lecture à mesure qu'il profère en imagination les mots qu'il lit, il est tout aussi impossible d'entendre les mots d'une langue connue sans aussitôt les comprendre. La lecture comme écoute et profération secrète se déploie en même temps que la lecture comme compréhension des significations et, plus que cela, l'atmosphère sonore, rythmique, affective, qui naît de l'imagination sonore du lecteur agit nécessairement sur la manière dont le lecteur comprend les significations attachées aux mots dont il goûte la saveur.

Dans les Recherches logiques, Husserl remarque ainsi que, hors d'une écriture visant l'univocité, certaines combinaisons de mots peuvent seulement produire une « aura de signification (Bedeutungshaufen) » au lieu d'une signification unique ${ }^{1}$. Mais à aborder la lecture du texte littéraire à l'aune des travaux d'Ingarden, il semble bien qu'il faille concevoir que l'émergence d'une aura de signification ne tient pas seulement à la structuration ellemême de la syntaxe. Elle tient aussi à l'attitude de lecture qui est mise en jeu et qui requiert, dans le cas de la lecture littéraire, de laisser émerger, précisément, ce qui vient nourrir en même temps que perturber la simple saisie des significations attachées aux vocables, à savoir l'atmosphère qui émerge d'un texte lu concrètement, dans l'épaisseur de sa texture sonore. Que les significations miroitent, pour le dire autrement, cela n'est pas seulement produit par la manière dont les significations sont mises en relation, logiquement, de manière plus ou moins lâche, mais cela naît de l'activité même de lecture, pour autant que l'écoute imaginaire est laissée à son efficacité et que l'imagination sonore du lecteur intervient ainsi dans la saisie des significations.

À cela, qui relève de l'effet de l'imagination du lecteur, comme profération intérieure du texte, sur la saisie des significations, il faut aussi ajouter une autre dimension par laquelle l'attitude de lecture continue d'introduire de l'indétermination dans le sens.

Ingarden souligne en effet que les mots sont des matériaux denses, chargés de toute l'épaisseur d'une culture et d'une histoire, alourdis de multiples connotations, complexifiés par la quantité d'usages dans lesquels

\footnotetext{
${ }^{1}$ E. Husserl, Recherches logiques II, t. 2, tr. H. Elie, A. Kelkel et R. Schérer, Paris, P.U.F, 1969, Quatrième Recherche, § 10, p. 110-111.
} 
ils ont déjà été rencontrés par le lecteur ${ }^{1}$. C'est cet arrière-fond, par lequel chaque mot est empesé de toute l'expérience que le lecteur possède de la langue du texte, qui est sollicité. C'est pourquoi Ingarden, afin de désigner les mots de l'œuvre littéraire, choisit de les dire vocables vivants ${ }^{2}$. Comme il le souligne lui-même à la fin de L'Euvre d'art littéraire, lorsqu'il en vient à parler de la « vie » de l'œuvre, ce qui caractérise avant tout le vivant, c'est sa capacité à se transformer continuellement, à préserver son identité sans demeurer le même ${ }^{3}$. Dès lors, l'activité de compréhension que le texte littéraire appelle exige une certaine souplesse par laquelle il s'agit de ne pas fixer trop vite le sens des mots - pour les laisser jouer ou parier, en tout cas, que le sens se nouera à partir du jeu entre les significations.

Pourquoi insinuer, néanmoins, que cette relation aux significations implique l'imagination du lecteur? Parce que celui-ci se doit d'anticiper les pistes - harmoniques, rythmiques, affectives, signifiantes - ouvertes par les mots, sans pourtant décider trop vite de la fixation d'un sens. Il doit se maintenir, pour ainsi dire, dans une attitude de disponibilité à l'égard des mots du texte, dont la teneur de sens ne peut être explorée que progressivement et à l'occasion de multiples mouvements d'après-coup, parce qu'elle se détermine dans leurs relations réciproques. Bref, il se doit, au fur et à mesure de la lecture, d'aborder sa performance de compréhension comme une performance possible et provisoire, précaire, en même temps qu'il doit être prêt à modifier son jeu si cela s'avère plus pertinent ou plus fécond. Aucun acte de compréhension, en cela, ne devrait être posé comme définitif ou, pour le dire autrement, le lecteur doit maintenir ses actes de compréhension dans une conscience non positionnelle.

En insistant sur la créativité de la langue tout autant que sur le poids des mots, Ingarden fait donc signe, en creux, vers la nécessaire souplesse et la nécessaire dextérité du lecteur. Le texte, comme une partition, en appelle à une réception active, par laquelle le lecteur accepte d'explorer de multiples

\footnotetext{
${ }^{1}$ R. Ingarden, L'Euvre d'art littéraire, op. cit., § 10, p. 53. C'est ainsi que certains mots nous paraissent - dans le cadre d'une même langue et d'une même communauté de vie - particulièrement aptes à comporter une certaine signification, et à n'être pas simplement d'emblée "compréhensibles », mais à porter également en eux diverses possibilités d'utilisation toutes particulières.

${ }^{2}$ Ibid., § 10, p. 53. Ingarden distingue vocables inertes et vocables vivants. Il faut entendre par vocables inertes l'ensemble des termes qui «s'épuisent dans leur signification claire, univoquement déterminée »; ce dont il est question, c'est en particulier des termes qui constituent les terminologies techniques et scientifiques.

${ }^{3}$ Ibid., $\S 64$, p. 291.
} 
pistes afin de trouver celle par laquelle il parviendra à comprendre quelque chose qui sonne juste.

\subsection{L'imagination comme production d'images : lecture et présentification}

Bien qu'elle laisse déjà apercevoir sa complexité, l'expérience de la lecture littéraire ne saurait s'en tenir à la lecture du texte comme expérience entrelacée des deux strates des vocables et des significations. Corrélativement, les actes d'imagination impliqués dans la lecture ne se réduisent pas, d'une part, à la profération intérieure du texte et, d'autre part, à la neutralisation de la compréhension même, dont le lecteur doit tenir qu'elle n'est jamais que provisoire. Un autre moment est essentiellement impliqué dans la lecture, qui consiste à se tourner vers ce que les significations, notamment nominales, désignent ou projettent en avant d'elles-mêmes. Le lecteur, attentif aux vocables et à leur saveur sonore et, dans le même mouvement, attentif aux significations, est aussitôt invité, par celles-ci, à viser à travers le texte ce qu'elles projettent, voire à effectuer le remplissement intuitif correspondant. Pour le dire brièvement, le lecteur ne saurait lire sans présentifier ce dont le texte parle.

Comme l'écrit Ingarden, chaque phrase, tissant ensemble des significations, accomplit le "déploiement nominal-verbal d'un "état-de-chose" " ${ }^{1}$. Mais la phrase, pour le lecteur qui se livre à l'expérience de lecture littéraire, ne se contente pas de posséder une signification discursive. Le remplissement des significations est motivé par la compréhension logique des phrases, voire le passage est exigé de la signification à ce qu'elle vise et offre de constituer dans l'intuition, afin que puisse advenir la strate des objets. Dire qu'un passage a lieu de la saisie des significations à la visée de ce qu'elles signifient, c'est précisément dire que les significations ne sont pas purement et simplement comprises mais qu'elles sont mises en fonction ${ }^{2}$. La saisie des significations, dans la configuration provisoire opérée par le lecteur, se joue comme le point de départ d'un mouvement intentionnel qui le porte auprès de cela même qu'elles projettent en termes de sens objectif. La compréhen-

\footnotetext{
${ }^{1}$ Ibid., $\S 19$, p. 109.

${ }^{2}$ Ibid., $\S 13$, p. 67 : « Cette mise-en-fonction conduit à son tour à ce que l'objet de référence correspondant, cohérent à la signification du mot, soit visé, ce qui permet aux autres couches de l'œuvre littéraire de parvenir au dévoilement ».
} 
sion des unités de sens conduit ainsi à la projection des objets intentionnels correspondants ${ }^{1}$.

Dans la perspective adoptée par Ingarden, l'imagination du lecteur qui intervient ici à même la mise en fonction des significations, pour viser ce qu'elles disent et désignent - , doit continuer d'être fermement guidée par le texte. L'imagination présentifiante qui intervient ici pour constituer la strate des objets ne joue pas librement mais se doit de laisser peser sur elle le poids des deux strates déjà abordées : celle des significations, qui se doivent d'être justement comprises, celle, surtout, des vocables, qui continuent de solliciter une imagination sonore et rythmique. Parce que les unités de sens sont toujours saisies comme l'envers de la texture sensible du texte, les états de choses sont visés et projetés dans un nimbe de qualités affectives nées de la musique du texte. Ainsi, parce que l'œuvre est l'articulation de ses strates et leur constitution entrelacée, viser ce dont le texte parle, ce n'est jamais perdre de vue le texte, mais voir à travers lui, au dedans de lui, ce qu'il donne à voir ${ }^{2}$.

À dire cela, on semble cependant aborder la constitution de la strate des objets moins comme un moment impliqué, aux côtés des autres, par la lecture, que comme un passage de seuil, après lequel la lecture se métamorphose pour perdre de vue le texte au profit du monde qu'il ouvre. Est-ce le cas? La constitution de la strate des objets signe-t-elle le déferlement d'une imagination visuelle, qui serait susceptible d'emporter la lecture?

\footnotetext{
${ }^{1}$ R. Ingarden, The Cognition of the Literary Work of Art, tr. fr. Crowley R. A et Olson K. R, Evanston, Northwestern University Press, 1973, § 9, p. 37.

${ }^{2}$ Nous n'avons pas le temps de développer cela ici, mais il faut néanmoins remarquer rapidement que la constitution de la strate des objets ne se joue pas comme succession d'objectivations définitives. Chaque objectivation est incessamment rejouée parce que les divers objets et états de choses signifiés gagnent de nouvelles déterminations, tout autant qu'ils peuvent en perdre d'autres, au fur et à mesure de la lecture. «The objectivities portrayed in a literary work (people, things, processes, events) are not in general immutable and are usually not portrayed in just one temporal phase or in one state but often have very involved fates, participate in various events, and sometimes undergo quite extensive transformations. All this is shown in a multiplicity of states of affairs which portray the same objects in various successive phases. Consequently, the objectification does not end with a constitution of the object in one phase of its being; rather, it is, or at least can be, carried out anew after each new event, after every transformation of the object. » (Ingarden, The Cognition of the Literary Work of Art, op. cit., § 10, p. 46). C'est ainsi que la constitution des divers objets et états de choses participant de la strate des objets s'élabore dans le temps long de la lecture - et dépend, pour cela, des variations d'attention du lecteur et de sa capacité à retenir les phases précédentes de sa lecture.
} 
La constitution de la strate des objets implique que le lecteur vise les multiples états-de-choses signifiés par le texte. Mais, si elle s'en tenait à cette opération, l'imagination du lecteur réaliserait une pluralité discrète d'actes de présentification, qui ne donnerait même jamais lieu à l'intuition d'un seul objet. En effet, le texte ne décrit jamais entièrement ce qu'il signifie ${ }^{1}$, ce qu'Ingarden ne cesse de souligner, en indiquant que le texte littéraire est plein de lieux d'indétermination: "We find such a place of indeterminacy wherever it is impossible, on the basis of the sentences in the work, to say whether a certain object or objective situation has a certain attribute $»^{2}$. Telle qu'Ingarden la conçoit, l'œuvre littéraire est donc une formation schématique, telle que les significations inscrites dans le texte ne disent pas tout de ce qu'elles invitent à présentifier ${ }^{3}$.

Or, le lecteur qui ne se tient pas devant le texte dans une attitude de spécialiste - qui ne procède pas à une lecture analytique dans laquelle il s'agit de s'en tenir au texte, pour le connaître - remplit nécessairement les lieux d'indétermination, c'est-à-dire accomplit de multiples actes d'imagination par lesquels il présentifie aussi, au moyen d'intentions qu'il forme en propre, ce que le texte ne dit pas. L'imagination du lecteur, pour autant qu'elle ne projette jamais simplement les déterminations signifiées par le texte mais, plus que cela, des objets et leurs horizons, voire un monde, opère donc en excès. Elle ne peut intervenir, simplement, pour donner un remplissement intuitif aux phrases du texte. Dès lors, il faut concevoir que l'imagination du lecteur, lorsqu'elle intervient, ne saurait être simplement guidée par le texte: elle doit proposer un surplus voire elle ne peut faire autrement. Le lecteur met en jeu des intentions qui excèdent la simple mise en fonction des significations inscrites dans le texte et par elles, il s'engage hors du sillon proposé par les phrases, vers les horizons de celles-ci. C'est ainsi que chaque lecteur, à chaque lecture, augmente ce dont le texte parle de nouvelles déterminations tandis qu'il en occulte d'autres, à mesure qu'il projette de nouveaux états de choses ainsi que leurs horizons.

Alors que l'imagination, telle qu'elle est mise en jeu dans les deux premières strates, relève d'un effort de coller au texte, de l'accompagner ou

\footnotetext{
${ }^{1}$ Cela, néanmoins, n'est pas propre au texte littéraire mais à l'expression en général, si, comme l'écrit Merleau-Ponty, "l'idée même d'une expression adéquate, celle d'un signifiant qui viendrait couvrir exactement le signifié, celle d'une communication intégrale, sont inconsistantes » (La Prose du monde, Paris, Gallimard, 1969, p. 42).

${ }^{2}$ R. Ingarden, The Cognition of the Literary Work of Art, op. cit., § 11, p. 50.

${ }^{3}$ R. Ingarden, L'Euvre d'art littéraire, op. cit., § 38, p. 211.
} 
de le faire résonner dans la complexité de ses harmoniques, il semble que l'imagination, telle qu'elle est requise pour la constitution de la strate des objets, implique un mouvement contraire. Elle semble prendre de l'élan au risque de creuser, aussi, la distance entre ce que signifie le texte et ce qu'expérimente, par la présentification, le lecteur. La constitution de l'œuvre littéraire advient donc comme la présentification d'un monde qui se trouve, dans l'exercice de chaque lecture, à la fois en excès et en défaut sur les déterminations inscrites dans le texte : le lecteur ne remplit pas toutes les significations - il se contente d'en saisir certaines seulement discursivement - tandis que le remplissement de certaines autres implique la mise en fonction d'intentions surnuméraires, pour le dire ainsi. Dans ce mouvement, c'est toute l'expérience du lecteur qui est mobilisée : celle, certes, qu'il a de son monde environnant et qui est susceptible d'être refigurée dans l'exploration de l'œuvre littéraire, mais aussi toute son expérience de lecteur et ses connaissances, notamment historiques, qui interviennent pour nourrir, avec plus ou moins de précision, le monde qui vient à figuration au cours de la lecture.

\subsection{La strate des aspects ou le secret de chaque lecture}

$\mathrm{Au}$ fur et à mesure que le lecteur constitue la strate des objets, en présentifiant nécessairement, aussi, ce que le texte ne signifie pas ou ne fait qu'indiquer, il fait advenir ce qu'Ingarden identifie comme la quatrième et dernière strate de l'œuvre, à savoir celle des aspects. Cette strate est peut-être la plus délicate à aborder, voire la plus problématique puisque les aspects, en effet, ne sont rien qui appartienne au texte ou que le texte signifie. Les aspects ne sont autre chose que les apparitions dans lesquelles le lecteur fait venir à la présence le monde ouvert par l'œuvre : ils ne sont pas ce qui est présentifié (objets et états-de-choses), mais la texture même, propre à chaque lecture, de la présentification, la dimension phénoménologiquement la plus concrète de la lecture. Pour le dire encore autrement, les aspects, contrairement aux objets et états-de-chose, ne sont pas signifiés par le texte : ils sont seulement motivés par la lecture. En effet, si le texte signifie ce qui doit être présentifié, il ne signifie pas toujours comment cela doit l'être, c'est-à-dire, concrètement, quelles apparitions il s'agit de faire advenir. C'est là la part du lecteur.

Or, comme le remarque Ingarden, le lecteur ne choisit pas les aspects qui émergent au cours de la lecture, en même temps qu'il fait advenir la strate des aspects selon les exigences de sa sensibilité propre: c'est ainsi qu'un certain lecteur laissera se déployer les aspects visuels du monde ouvert 
par l'œuvre, là où un autre s'absorbera plutôt dans la dimension sonore ou tactile de ce dernier. Certes, le texte peut guider cela ${ }^{1}$ et fournir à son lecteur une foule d'éléments qui nourrissent son imagination et imposent, pour le dire ainsi, certaines apparitions. Mais, même dans ce cas, comme y insiste Ingarden, la constitution de la strate des aspects ne peut que laisser la place aux habitus de perception du lecteur, à ses désirs, à ses rigidités associatives. C'est ainsi que, dans une certaine mesure, la constitution de la strate des aspects ne se dit que difficilement constitution - elle ne semble pas relever, en tout cas, d'une synthèse active, mais, bien plus, de la manière dont chaque lecteur est affecté par le texte et lui répond de manière essentiellement passive.

Ce qui apparaît ici, c'est la possibilité d'une tension entre ce que le texte autorise et ce que le lecteur accomplit, entre les horizons ouverts par le texte - l'implicite que le lecteur est invité à présentifier aussi - et l'invention pure et simple de nouvelles déterminations « qui ne s'accordent pas avec les moments objectaux positivement déterminés par celui-ci [le texte] ${ }^{2}$. Si l'efflorescence des aspects est requise, elle peut donc aussi submerger la lecture et comme contrarier à rebours la constitution de la strate des objets, voire la compréhension des significations inscrites dans le texte. L'imagination du lecteur peut larguer les amarres, s'emporter, de telle sorte que la constitution de l'œuvre peut céder le pas à un éloignement de celle-ci, voire à une occultation, le monde fantasmé par le lecteur venant recouvrir celui qui pourrait être présentifié en se laissant plus fermement guider par le texte.

C'est en remarquant ceci qu'Ingarden se trouve conduit à distinguer entre des concrétisations adéquates de l'œuvre qui, bien que sollicitant l'imagination libre du lecteur, acceptent d'être guidées par le texte, et des concrétisations inadéquates, ou arbitraires pourrait-on dire, qui perdent de vue le texte pour projeter un monde qui ne lui est plus relié que par quelques fils.

\footnotetext{
${ }^{1}$ Ibid., § 12, p. 61 : «Les œuvres dans lesquelles les aspects s'imposent au lecteur avec une telle force de suggestion seront bien plus fidèlement reconstruites dans leur strate d'aspects que les œuvres dans lesquelles les aspects sont déterminés idealiter et reliés aux choses figurées sans qu'ils s'imposent au lecteur. Les œuvres dans lesquelles les éléments intuitifs des aspects disponibles dans les différents domaines de l'expérience sensible sont très riches peuvent être incorrectement reconstituées par le lecteur, si le lecteur ne parvient pas à rendre justice à la multiplicité des aspects. »

${ }^{2}$ Ibid., § 38, p. 214. Nous précisons.
} 


\section{L'œuvre et ses concrétisations : la pluralité des lectures}

En revenant, certes rapidement, sur la pluralité des strates de l'œuvre littéraire telles qu'Ingarden les identifie, et en articulant cette ontologie de l'œuvre d'art à une esquisse de phénoménologie de la lecture — dans laquelle il s'agissait d'indiquer quels actes d'imagination étaient requis par chaque strate, ainsi que la manière dont ces actes s'entrelacent les uns aux autres et se motivent mutuellement -, nous avons aperçu la complexité de l'expérience de lecture, corrélative de la complexité de l'œuvre.

Celle-ci exige du lecteur qu'il se tienne en même temps à plusieurs niveaux d'expérience et surtout qu'il s'efforce de tenir ensemble les multiples strates de l'œuvre, sans en négliger aucune ou en favoriser aucune, tout en étant attentif à leur équilibre mutuel et à leur harmonie. Bref, l'œuvre exige du lecteur des mouvements contraires, une sorte de distorsion, pourtant harmonieuse ou cohérente, de l'attention, qui requiert une mise en œuvre différenciée de l'imagination - comme profération et écoute intérieure, comme ouverture de possibilités, comme attention neutralisée, comme présentification, et presque, in fine, avec la strate des aspects, comme rêverie libre ou passive. C'est bien parce que chaque lecteur articule toutes ces dimensions, à chaque fois comme il le peut, que les multiples lectures d'un même texte ne donnent jamais lieu à la même expérience.

C'est d'ailleurs pour cela qu'Ingarden, prenant en vue cette pluralité nécessaire, ne parle pas d'actualisation de l'œuvre par le lecteur, mais de concrétisations de l'œuvre littéraire, en désignant, par-là, la manière même dont chaque lecteur, à chaque fois et selon ses ressources, donne vie à celleci. À chacune de ses lectures, l'œuvre est concrétisée de manière singulière : les vocables sont lus selon un certain rythme, un certain ton; une certaine voix est projetée qui accompagne la lecture du texte; les états de choses projetés par les phrases donnent lieu à des objectivations qui dépendent des foyers d'attention du lecteur. Et, de plus, à chacune des lectures, sont motivées certaines apparitions qui ne sont essentiellement pas prescrites par le texte. C'est en cela que l'œuvre littéraire, si on l'entend bien comme expérience de la lecture du texte, a une vie: elle est en mouvement, elle se modifie sans cesse, au fil des lectures qui sont faites d'elles ${ }^{1}$.

Que les lectures soient plurielles, cela ne signifie cependant pas qu'elles sont toutes valables. L'ontologie de l'œuvre littéraire et la phénoménologie d'Ingarden sont en effet sous-tendues par une esthétique qui s'efforce de formuler les critères d'une bonne ou juste lecture. Cela s'est

${ }^{1}$ Ibid., p. § 64, p. 293. 
manifesté, d'ailleurs, dans la partie précédente, dans la manière même dont nous avons pu rendre compte de l'approche d'Ingarden qui choisit toujours de décrire ce qui doit se passer : non seulement ce qui appartient à la légalité phénoménologique de l'expérience de lecture mais aussi ce qui est exigé pour que l'expérience de lecture soit valable ${ }^{1}$. Dans cette articulation de la réflexion esthétique à la description phénoménologique se met en place une tension qui permet d'explorer plus avant la manière dont Ingarden conçoit la participation de l'imagination à la lecture.

\subsection{L'œuvre et ses concrétisations: jusqu'aux limites d'une simple phéno-} ménologie de la lecture?

In reading, there are a multiplicity and variety of experiences and acts of apprehension which the reader must perform almost simultaneously if the reading is to do even approximate justice to the many strata and the many forms of literary phenomena in one and the same literary work of art. As a result, the reader does not give the same active attention to all the acts he performs, nor does he perform all of them with the same requisite vividness or thoroughness. Consequently, many details in the various strata and phases of the work undergo more or less significant distortion in concretization. Many details of the structure of the work may be omitted or incompletely constituted in concretization; or else they may be overconstituted, developed too prominently; or they may even be falsified ${ }^{2}$.

À suivre Ingarden dans sa conception de l'œuvre d'art et, corrélativement, de l'expérience de lecture, il faut admettre qu'aucun lecteur ne saurait constituer parfaitement l'œuvre littéraire, de sorte que toute lecture, malgré toutes les ressources imaginatives qu'elle mobilise et les excès qu'elle contient, est essentiellement en défaut. La complexité de l'œuvre littéraire et corrélativement de l'expérience de lecture est telle que, selon Ingarden, le lecteur « est surchargé, et de ce fait ne peut vivre unitairement toutes les composantes de cette saisie globale $»^{3}$. Chaque expérience de lecture est une expérience lacunaire, dans laquelle il est par principe impossible que toutes les dimensions de l'œuvre soient pleinement constituées par le lecteur. En d'autres

${ }^{1}$ Comme l'écrit Walter H. Clark dès la première phrase de son compte rendu de The Cognition of the Literary Work of Art, « in simplest terms, though it is not simple, this is a book about how to read" (The Journal of Aesthetics and Art Criticism, Vol. 33, No. 2, Winter, 1974, p. 220).

${ }^{2}$ R. Ingarden, The Cognition of the Literary Work of Art, op. cit., § 14, p. 90.

${ }^{3}$ R. Ingarden, L'CEuvre d'art littéraire, op. cit., § 62, p. 283. 
termes encore, l'expérience de lecture est telle qu'en elle, l'œuvre n'est jamais saisie que dans ce qu'Ingarden nomme justement un «raccourci perspectiviste », dans lequel non seulement ce ne sont que certains moments passés et à venir de la lecture qui sont présents en rétentions et protentions, mais ce ne sont aussi que certaines strates qui sont placées au-devant de l'attention pendant que d'autres sont repoussées à l'arrière-plan. Dans une certaine mesure, la phénoménologie de la lecture d'Ingarden est d'abord une phénoménologie de l'échec de la lecture, de l'impossibilité essentielle où se trouve le lecteur d'éprouver et de tenir ensemble la totalité de l'œuvre.

Pourtant, lorsqu'Ingarden aborde, dans la dernière partie de L'Euvre d'art littéraire, ce qu'il nomme la vie des œuvres, il remarque aussi que lors de la lecture, à même l'expérience de lecture et donc la rencontre singulière d'un texte et d'un lecteur, on ne saurait observer aucune lacune, aucune indétermination, aucune potentialité ${ }^{1}$. Autrement dit, pour chaque lecteur, à chaque fois, il ne manque rien à son expérience en même temps que sa lecture n'est pas vécue comme une lecture singulière, partielle ou insuffisante. Dire cela, c'est comprendre que le lecteur vit dans la concrétisation qu'il opère de l'œuvre, qu'il vit en elle à mesure qu'elle advient, et qu'il vit en elle sa rencontre avec l'œuvre elle-même. Ce n'est en effet que depuis un point de vue théorique et surplombant, ou encore après-coup, que l'on peut distinguer entre l'œuvre et ses concrétisations, par lesquelles elle est rendue vivante et présente par ses lecteurs.

Comme Ingarden le souligne lui-même dans son article de 1969, «De l'esthétique phénoménologique, essai de définition $»^{2}$, une des lignes directrices de son approche de l'œuvre d'art consiste effectivement à mettre en avant l'importance des concrétisations de l'œuvre d'art littéraire, par lesquelles celle-ci prend à chaque fois vie dans l'expérience de ses lecteurs. L'œuvre, exclusivement accessible à travers ses concrétisations, qui articulent les quatre strates présentées plus haut, ne se confond donc aucunement avec le texte. Elle inclut la participation du lecteur :

En tant que formation commune de l'artiste et de l'observateur, une concrétisation diffère d'une autre concrétisation selon des degrés plus ou moins grands, mais la nature et l'étendue des variations dépendent à la fois des caractères de l'œuvre (en particulier du type d'art auquel elle appartient) et de la compétence de l'observateur, comme aussi de la nature empirique de son

\footnotetext{
${ }^{1}$ Ibid., $\S 61$, p. 281.

${ }^{2}$ R. Ingarden, «De l'esthétique phénoménologique, essai de définition» (1969), in Esthétique et ontologie de l'œuvre d'art: choix de textes 1937-1969, tr. fr. Patricia Limido-Heulot, Paris, Vrin, 2011, p. 45.
} 
appréhension [Erfassung $]$ et des conditions particulières dans lesquelles elle a lieu ${ }^{1}$.

On aperçoit ici une des difficultés qui guettent l'approche d'Ingarden, dans la mesure où l'on semble bien proche d'une position qui, parce qu'elle décrit la manière dont l'œuvre est nécessairement prise dans des expériences et des concrétisations diverses et contingentes, rendrait possible l'affirmation selon laquelle l'œuvre, dans les diverses strates qui la composent, donne lieu à une pluralité indéterminée de concrétisations par lesquelles elle se trouve non seulement expérimentée par les lecteurs, mais aussi appropriée voire altérée. Il semble bien, de notre point de vue, que c'est cela qui doit être conclu si l'on en reste à une simple description phénoménologique de l'expérience de lecture, qui donne toute sa place à l'activité du lecteur et à l'immixtion, dans la constitution même de l'œuvre, de ses compétences linguistiques, de son expérience passée et de son imagination.

Cependant, pour Ingarden, cela qui ressortit à une analyse phénoménologique de l'expérience de lecture ne suffit pas ; ou, pour le dire autrement, son propos ne saurait se tenir dans les limites d'une phénoménologie. Qu'il choisisse de nommer sa démarche une esthétique phénoménologique manifeste la singularité de sa position, tout autant que la tension qui traverse son travail : en effet, si chaque lecteur concrétise l'œuvre à sa manière, s'il n'y a pas de lecture totale, s'il est donc seulement possible d'observer, à même sa propre expérience, des lectures imparfaites, la description que propose Ingarden, qui ne cesse de dire ce qui doit ou devrait se passer, ne cesse de tourner le regard vers une lecture idéale. Il fait jouer, ce faisant, à même la description phénoménologique de l'expérience de lecture, l'idée d'une lecture parfaitement accomplie - lecture impossible dont l'idée travaille la description, contribuant ainsi à introduire de la prescription, de la normativité. À la description de l'expérience de lecture, telle qu'elle est motivée par le texte, vient s'ajouter une recherche des critères par lesquels il est possible d'identifier une bonne lecture ou encore la constitution de concrétisations valables.

Ingarden, en effet, ne peut se contenter d'affirmer, comme conséquence nécessaire de sa description phénoménologique de l'expérience de lecture, la pluralité des expériences de lecture et, par suite, la nécessaire appropriation de l'œuvre par les lecteurs qui la concrétisent. Dire purement et simplement cela, ce serait, en effet, depuis une phénoménologie, renoncer à

${ }^{1}$ R. Ingarden, «Valeurs esthétiques et valeurs artistiques», in Esthétique et ontologie de l'œuvre d'art : choix de textes 1937-1969, op. cit., p. 143. 
toute évaluation de la lecture et faire place à tous les lecteurs possibles. Mais Ingarden refuse de s'en tenir à une pure phénoménologie de la lecture, qui prendrait acte de la légalité de l'expérience de lecture littéraire en remarquant que celle-ci inclut essentiellement dans sa structure l'intervention de l'imagination du lecteur et, parce que cette imagination est libre, l'immixtion de son imaginaire. L'analyse phénoménologique, pour ce qui intéresse Ingarden, ne suffit pas dans la mesure où elle permet seulement de dire ce qui se passe pour tout lecteur : paradoxalement, c'est la dimension eidétique de l'analyse phénoménologique, qui décrit les coordonnées essentielles de l'expérience, qui signe les limitations de sa pertinence. Il ne suffit pas, pour Ingarden, de décrire ce qui se passe pour qu'un lecteur comprenne quelque chose en lisant, se figure quelque chose en lisant, éprouve quelque chose en lisant - mais il faut aussi se mettre en mesure de saisir comment un lecteur peut comprendre adéquatement, se figurer adéquatement, ressentir adéquatement. Ingarden, scrutant les multiples dimensions de la lecture, faisant ressortir, à chaque fois, les manquements possibles du lecteur, insiste sur le fait que, parmi ces concrétisations - dont une phénoménologie n'aurait qu'à attester l'efflorescence - certaines sont plus valables que d'autres. Bref, certaines concrétisations sont adéquates, tandis que d'autres ne le sont pas; ce qui implique aussitôt que certains lecteurs savent faire advenir l'œuvre tandis que d'autres ne peuvent que la trahir.

Dès lors, ce qui joue, ainsi, dans l'esthétique phénoménologique d'Ingarden, ce n'est pas seulement l'idée d'une lecture parfaite mais surtout, pour fonder l'exclusion de certaines pratiques comme impropres à constituer l'œuvre littéraire, l'image du bon et du mauvais lecteur.

\subsection{Concrétisations inadéquates et excès d'imagination}

Toute lecture, pour autant qu'elle est une lecture vivante et non une lecture analytique ${ }^{1}$, requiert l'intervention de l'imagination du lecteur, avec tout ce qu'elle porte aussi de l'époque culturelle à laquelle il appartient ${ }^{2}$. Cette imagination est sollicitée, notamment, lors du remplissement des lieux d'indétermination, de telle sorte que la participation du lecteur à l'œuvre se joue bien comme un ajout, dans lequel le lecteur apporte ce que le texte ne

\footnotetext{
${ }^{1}$ R. Ingarden, The Cognition of the Literary Work of Art, op. cit., § 11, p. 52. La lecture analytique est notamment celle qui résiste à remplir les lieux d'indétermination pour les considérer comme tels, afin de connaître l'œuvre dans sa structure.

${ }^{2}$ Ibid., § 11, p. 55.
} 
contient pas. Ces déterminations supplémentaires voire excédentaires, Ingarden le souligne, relèvent, le plus souvent, de la strate des objets figurés : l'imagination du lecteur, libérée de toute entrave, joue à plein dans la figuration du monde proposé par le texte littéraire, de telle sorte qu'il faut concevoir ce qui flotte dans la tête du lecteur comme l'émergence d'un monde qui est co-porté par le texte et le lecteur et donc comme co-créé par ce dernier qui apporte les ressources de son imagination ${ }^{1}$.

Cependant, parce que l'immixtion d'une imagination présentifiante est requise par l'œuvre si elle doit être constituée, le fait de lire avec l'imagination, en se figurant ce qui est signifié par le texte, n'est pas le propre d'une lecture naïve mais bien une dimension essentielle de toute lecture nonanalytique —c'est-à-dire de toute lecture qui se joue comme contact avec $l^{\prime} œ u v r{ }^{2}$ et non comme connaissance du texte. Bref, le partage entre bonne et mauvaise lecture ne saurait reposer sur l'intervention ou non de l'imagination comme faculté de présentification. Il repose, cependant, sur un examen de la manière dont l'imagination, en ce sens-là, est mise en jeu et, plus précisément, de la manière dont la constitution de la strate des objets et des aspects parvient à demeurer contrainte par les strates des significations et des vocables.

En quoi consiste, alors, une mauvaise lecture ? Ou encore, comment une concrétisation inadéquate se forme-t-elle?

Lorsqu'il fait intervenir, dans son travail, l'image du mauvais lecteur, Ingarden décrit celui-ci comme un lecteur qui vient à l'œuvre pour son propre divertissement et, surtout, comme un lecteur qui, plutôt que d'être attentif aux diverses strates de l'œuvre, est accaparé par l'intrigue, sa progression et sa résolution :

I shall not occupy myself further here with how literary work of art come to be known by readers who merely want to amuse themselves without caring what is really which gives them enjoyment. Since they undoubtedly read, they probably have to perform the majority of the cognitive acts which we tried to describe above. But they do it merely fleetingly, imprecisely and idly, simply in order to progress and to find how the story ends. They look up the « happy ending $»^{3}$.

\footnotetext{
${ }^{1}$ Ibid., § 11, p. 53.

${ }^{2}$ Ibid., § 12, p. 56-57. Ingarden insiste bien sur le fait que les aspects doivent devenir présents (actual) pour le lecteur, de telle sorte que ce qui est signifié par le texte atteint, au cours de la lecture, une présence intuitive.

${ }^{3}$ Ibid., $\S 23$, p. 172.
} 
Le mauvais lecteur, c'est donc celui qui accomplit très sûrement toutes les opérations décrites plus haut, celles-ci étant essentielles à l'expérience de lecture, mais qui les accomplit avec désinvolture. Nous l'avons dit, aucune lecture ne saurait concrétiser parfaitement et définitivement l'œuvre : l'imperfection de la concrétisation réalisée ne saurait donc être un critère de partage entre bon et mauvais lecteur. Ce qui distingue ces derniers, semble-til, c'est l'attitude avec laquelle ils viennent au texte voire l'intention avec laquelle ils lisent.

Le bon lecteur lit pour faire venir à la présence l'œuvre d'art du mieux qu'il peut, autrement dit, il travaille pour elle, il se met à son service, tandis que le mauvais lecteur est celui qui use de l'œuvre pour son propre plaisir. Plus précisément encore, le mauvais lecteur est celui dont l'attention est fuyante, pour le dire ainsi : il n'est pas attentif à chacune des strates et aux relations réciproques de celles-ci, mais il les traverse toutes pour être en contact avec la seule chose qui l'intéresse, à savoir l'intrigue ${ }^{1}$. Dans cette lecture-là, où l'intérêt est tout entier tourné vers ce qui est raconté, les quatre strates - les vocables, les significations, les objets, et les aspects figurés par le lecteur - sont ravalées au rang de moyens pour accéder à l'intrigue. Le mauvais lecteur est celui qui, loin de viser, par son activité de lecture, une «concrétisation fidèle de l'œuvre (faithful concretization) » ${ }^{2}$, dans une attitude de responsabilité devant l'œuvre, ne se préoccupe que de son propre plaisir. Tel qu'Ingarden le décrit, il est ainsi prêt à opérer toutes les distorsions nécessaires dans la concrétisation de l'œuvre, pour autant qu'il atteint son but, c'est-à-dire qu'il retire de l'utilisation de l'œuvre le plaisir recherché.

Le partage entre bon et mauvais lecteur ne repose donc pas d'abord sur des critères de dextérité technique. Mal lire, avant toute chose, ce n'est pas ne pas savoir lire, ne pas savoir tenir ensemble les strates de l'œuvre par exemple; mais le partage est bien plus un partage moral, qui sépare les lecteurs responsables, venant à l'œuvre pour lui rendre justice, et les lecteurs injustes qui usent de l'œuvre comme un moyen pour leur propre amusement. Or, selon Ingarden, il s'agit là de la majorité des lecteurs.

\footnotetext{
${ }^{1}$ Il est dès lors à remarquer qu'Ingarden, dont le travail porte sur l'œuvre littéraire en général, lorsqu'il fait surgir la figure du mauvais lecteur, parce qu'il met en avant l'intérêt pour l'intrigue, dessine en fait la figure du lecteur de roman. Le mauvais lecteur est celui qui consomme des romans.

${ }^{2}$ R. Ingarden, The Cognition of the Literary Work of Art, op. cit., § 23, p. 172.
} 
The preponderant majority of readers, to be sure, belongs to this type of " consumers ", but they can be of interest only to the psychology or the sociology of the mass consumption of art. They play no role for our purposes, except, perhaps, at most as an example of how literary works of art should not be read, since such a reading does them an injustice ${ }^{1}$.

La tension précédemment évoquée, entre esthétique et phénoménologie, est ici manifeste. Il pourrait sembler, en effet, que l'enjeu d'une phénoménologie de la lecture littéraire est de décrire ce qui se passe nécessairement pour tout lecteur. Or, pour Ingarden, il s'agit au contraire d'exclure du champ de vision la majorité des lecteurs, indignes d'une enquête philosophique. La description prend alors un autre sens et n'en devient que plus problématique : soit elle est description de l'expérience d'une minorité de lecteurs identifiés comme compétents - d'une élite ; soit elle est description, hors sol empirique, de ce que devrait être idéalement la lecture d'une œuvre littéraire. Dans ce mouvement où les réquisits de la méthode phénoménologique semblent particulièrement bouleversés, se glisse aussi un manifeste mépris de classe et par suite l'exclusion des simples lecteurs, identifiés comme sujets indignes des discours philosophiques - puisqu'il sont réservés à la psychologie ou à la sociologie des masses et donc, dans le même mouvement, pathologisés. La condamnation morale des mauvais lecteurs, irresponsables jouisseurs, est aussi leur identification comme lecteurs populaires, sauvages ou anormaux - étrangers aux normes de l'appréciation esthétique des œuvres.

\subsection{Le bon lecteur et le sens de la mesure}

Si le mauvais lecteur est un lecteur-consommateur, usant des œuvres pour son propre plaisir - et pour un plaisir répétitif qui consiste, in fine, à se laisser fasciner par l'intrigue, en négligeant l'attention requise à l'œuvre ellemême dans la complexité de sa stratification, le bon lecteur, on l'a compris, n'est pas non plus le lecteur professionnel, le spécialiste de littérature, par exemple, qui vient aux œuvres dans un projet de connaissance de celles-ci. Le bon lecteur dont Ingarden vise à décrire - ou à prescrire - l'activité est l'amateur de littérature, l'esthète. Au regard de cela, l'approche d'Ingarden semble bien plus relever d'une esthétique du goût que d'une phénoménologie de la relation aux œuvres littéraires.

${ }^{1}$ Idem. 
Qui est le bon lecteur, alors, ou l'esthète, si l'on tente seulement de le décrire par contraste avec la figure du mauvais lecteur? Il est celui, certes, qui s'efforce de tenir ensemble les diverses strates de l'œuvre dans un mouvement dialectique: pour lui, la face sensible des mots ne s'abolit pas dans la compréhension des intentions de signification, qui elles-mêmes ne disparaissent pas devant cela même qu'elles désignent et invitent à présentifier $^{1}$. Il est donc celui qui se laisse guider, dans ses actes de présentification, par une attention soutenue accordée aux significations auxquelles il s'efforce, dans la mesure du possible, d'être fidèle - tout autant qu'aux halos affectifs qui naissent de la constitution de la strate des vocables. Il est donc un lecteur capable d'une attention plurifocale, pour le dire ainsi, et plus précisément, d'une attention tenue, consciemment entretenue. C'est aussi un lecteur qui accomplit des actes de présentification pertinents, c'est-à-dire qui fait venir le monde ouvert par l'œuvre à l'intuition en se fondant sur une compréhension fine de la strate des significations ${ }^{2}$, que les strates des objets et des aspects, alors, ne viennent pas trahir ou recouvrir mais bien rendre concrètes ${ }^{3}$. Bref, c'est un lecteur qui, dans l'œuvre d'art littéraire, demeure soucieux de l'art et vient à l'œuvre avec le désir d'éprouver ce que cette œuvre-ci, comme objet artistique, est capable de lui faire éprouver. Ce pour quoi Ingarden distingue entre les émotions auxquelles se livre le lecteur-consommateur, qui sont purement provoquées par l'intrigue, et les émotions esthétiques dont est capable le bon lecteur, ces émotions naissant de la relation à l'œuvre dans la saisie de sa complexité propre.

Nous sommes donc invités sur ce point à questionner plus avant l'imagination du lecteur et ses effets pour déterminer comment la possibilité d'éprouver ou non des émotions esthétiques est dépendante d'une certaine

\footnotetext{
${ }^{1}$ R. Ingarden, The Cognition of the Literary Work of Art, op. cit., §26, p. 227.

${ }^{2}$ Ibid., $\S 26$, p. 226.

${ }^{3} \mathrm{Ibid}, \S 28$, p. 308 : « The more regular, the more exact, the deciphering of the work, the more attentively the reader takes into consideration not merely the individual sentences but also the connection between them, defers to the suggestions of the text, and actively projects the portrayed world in intention in accordance with the meaning, at the same time concretizing the aspects under the influence of the poetic language and also removing, in conformity with the spirit of the work, exactly those places of indeterminacy which should be removed, the more probable it is that the aesthetic concretization of the work will fall within the range of concretization allowed by the work of art and, consequently, that the aesthetic concretizations of the same literary work of art will approximate one another. »
} 
discipline de l'imagination, apte à prévenir toute absorption « pathologique » dans l'intrigue.

\section{La délicatesse de l'imagination}

Patricia Limido-Heulot souligne qu'Ingarden déploie son explicitation des opérations par lesquelles l'objet esthétique est constitué

en martelant continûment que la condition principale pour que la concrétisation conduise à l'apparition de l'objet esthétique, c'est qu'elle soit menée dans l' " attitude esthétique » (Einstellung), ou mieux encore dans l'attitude esthétique « correcte», appropriée et adéquate ${ }^{1}$.

Comme elle l'indique, il s'agit par cette insistance, d'éviter de tomber, en même temps, dans les pièges du psychologisme et du relativisme, c'est-à-dire de réduire l'œuvre aux opérations subjectives du lecteur et de conclure, aussi, de la pluralité nécessaire des lectures à leur égalité de principe. L'insistance sur l'attitude esthétique adéquate permet donc de limiter les pouvoirs du lecteur ou plutôt d'exiger leur limitation. Il est un fait que le lecteur peut faire ce qu'il veut de l'œuvre ou la concrétiser sans savoir ce qu'il fait -, mais Ingarden, en réclamant une certaine attitude, soumet l'expérience de lecture à une morale qui en appelle à la responsabilité du lecteur pour l'œuvre ou à sa «bonne volonté » ${ }^{2}$.

Or, dans cette même introduction, Patricia Limido-Heulot souligne la tentation que l'on pourrait avoir de comparer Ingarden et Hume et elle rappelle notamment le passage qui, dans la « Norme du goût », convoque une anecdote tirée de Don Quichotte pour souligner la manière dont certains manifestent une certaine délicatesse de l'imagination qui les rend capables d'éprouver certaines émotions subtiles ${ }^{3}$. L'attention esthétique jouerait donc, chez Ingarden, le même rôle que la délicatesse du goût chez Hume, en permettant de dire de quel type d'attention l'esthète est capable : «l'attitude esthétique est celle qui, dans un effort subjectif d'acuité et de voir

1 P. Limido-Heulot, «L'esthétique phénoménologique de Roman Ingarden», introduction à Esthétique et ontologie de l'œuvre d'art: choix de textes 1937-1969, op. cit., p. 17.

${ }^{2}$ Ibid., p. 18.

${ }^{3}$ D. Hume, « De la règle du goût », Essais sur l'art et le goût, tr. fr. M. Malherbe, Paris, Vrin, 2010, p. 92. 
discriminant, cherche à se laisser saisir par l'objectité elle-même, se laisse fasciner, sidérer pour entrer dans une vue compréhensive $»^{1}$.

En suivant dans cette dernière partie la suggestion de Patricia LimidoHeulot, qui invite à comparer Ingarden et Hume, nous souhaitons insister une nouvelle fois sur les usages différenciés de l'imagination que font le bon et le mauvais lecteurs - tels qu'ils sont conçus par Ingarden - pour explorer plus avant la relation entre imagination et émotions telle qu'elle se noue lors de la lecture de l'œuvre littéraire. En relisant notamment Ingarden au regard de l'essai de Hume « De la délicatesse du goût et de la passion », il s'agira de distinguer entre usages esthétiques et non-esthétiques de l'imagination dans l'expérience de lecture.

\subsection{Délicatesse de la passion et consommation romanesque}

Au tout début de son essai, " De la délicatesse de la passion et du goût », Hume met en scène ceux qui, trop sensibles aux accidents de la vie, sont ballottés de passions heureuses en passions tristes. Suspendus aux coups du sort, ils éprouvent avec une intensité trop grande les émotions que font naître les circonstances et se trouvent, de fait, plongés dans une immense instabilité affective. Plus que cela, ces êtres délicats, « sujets à une grande délicatesse de la passion $»^{2}$, sont suspendus au comportement des autres à leur égard : il est en effet question, dans le texte de Hume, d'une sensibilité aux « faveurs» et aux «bons offices », ainsi qu'à l'« honneur » et aux « marques de distinction », tout autant qu'à l' « injustice » subie ou au « mépris » ${ }^{3}$.

Quelle relation y a-t-il alors entre ceux-ci et le mauvais lecteur d'Ingarden ? Ce dernier, nous l'avons indiqué, est intéressé au déroulement de l'intrigue, de telle sorte qu'il devient aveugle aux strates de l'œuvre littéraire perçues pour elles-mêmes et qu'il est prêt à les trahir pour maintenir un plaisir tout entier lié au suspense narratif. Mais Ingarden précise cela de la manière suivante : le lecteur-consommateur n'est ému par rien qui relève d'une appréciation esthétique de l'œuvre mais il est captivé par « the vicissitudes of the people portrayed in it $»^{4}$. Le mauvais lecteur, donc, c'est celui

\footnotetext{
${ }^{1}$ P. Limido-Heulot, «L'esthétique phénoménologique de Roman Ingarden », art. cit., p. 19.

${ }^{2}$ D. Hume, « De la délicatesse du goût et de la passion », Essais sur l'art et le goût, op. cit., p. 65.

${ }^{3}$ Idem.

${ }^{4}$ R. Ingarden, The Cognition of the Literary Work of Art, op. cit., § 25, p. 222.
} 
qui vient à une œuvre littéraire pour éprouver les émotions que l'on ressent au contact des autres et non des œuvres. Dans une certaine mesure, il est celui qui se trompe d'objet.

Ce parti-pris d'Ingarden qui consiste à exclure la relation aux personnages de l'expérience esthétique est actif dans son approche d'autres arts. Afin de cerner les enjeux de cette exclusion de l'intérêt pour la vie des autres et de l'empathie il est alors possible, par exemple, de mettre en vis-à-vis l'expérience non-esthétique de la lecture, ou la consommation littéraire, et la contemplation non esthétique d'un tableau.

Dans l'article de 1969 déjà cité, « De l'esthétique phénoménologique : essai de définition », Ingarden prend en effet le cas d'un spectateur qui, contemplant un tableau, parvient non seulement à appréhender certains objets à partir des taches colorées disposées sur la toile, mais se trouve aussi soudainement touché par «l'expression» de la personne figurée dans la tableau, par « une certaine humeur, une émotion ou un état psychique, ou bien un certain trait de caractère de la personne figurée ${ }^{1}$. Pour Ingarden lorsque cela advient - lorsque le spectateur est touché par la personne figurée en portrait ou lorsque le lecteur est touché par le personnage - le récepteur de l'œuvre d'art, qui a affaire à l'émotion figurée d'un autre luimême figuré, se trouve requis par ce qui se manifeste de telle sorte que son expérience se trouve suspendue à la relation qu'il parviendra à établir avec cette « expression» : soit il comprend cette expression, nous dit Ingarden, soit « il peut se trouver désagréablement touché ou plongé dans une atmosphère insolite $»^{2}$. Autrement dit, l'expérience qui a lieu au contact de l'œuvre d'art est traversée par des enjeux qui relèvent de relations interhumaines et de la manière dont le récepteur, indépendamment donc de toute compétence esthétique, est capable de plus ou moins d'empathie dans sa relation aux autres. Plus précisément, pour Ingarden, l'expérience faite au contact de l'œuvre est parasitée ou interrompue, puisque la réaction émotionnelle provoquée par l'expression perçue consiste pour lui en un élément « en quelque sorte extra-esthétique $»^{3}$. Cette réaction, qui met en jeu les habitus par lesquels le récepteur s'oriente dans ses relations avec les autres, relève de la manière dont il comprend ou ne comprend pas l'autre figuré - et les autres en général - et non de la manière dont il se tient, dans l'attitude esthétique, en relation avec l'œuvre.

${ }^{1}$ R. Ingarden, «De l'esthétique phénoménologique, essai de définition» (1969), art. cit., p. 59.

${ }^{2}$ Idem.

${ }^{3}$ Idem. 
Si la relation du lecteur aux personnages et aux situations figurées est problématique, lorsqu'elle barre la route aux émotions esthétiques, ce n'est pas là le seul danger qui guette la lecture. Pour Ingarden, en effet, le mauvais lecteur est aussi celui qui vient aux œuvres d'art pour enrichir sa vie ou éprouver un certain état de bien-être ${ }^{1}$. Si cette remarque rejoint le refus assumé par Ingarden de réduire l'expérience esthétique au plaisir qu'elle serait censée susciter, en même temps qu'elle rejoint son refus d'assimiler expérience esthétique et divertissement, elle semble aussi rejeter hors de l'expérience de lecture une dimension qui lui appartient de fait - et qui fonde, pour de nombreux lecteurs, le goût de la lecture ${ }^{2}$.

To the aesthetically naive person, who is primarily concerned with having certain experiences, it seems that the more he is moved by certain objects (especially by works of arts), and the more multifarious and lively his emotional life is, the more valuable are the objects of his experience, or, in other words, the more effective his aesthetic experience is ${ }^{3}$.

Certes, ces remarques font signe peut-être vers le développement de l'art kitsch, qui vise à séduire les lecteurs par une forme de sensationnalisme ${ }^{4}$; elles visent aussi les prises de positions irrationalistes qui survalorisent la place du sentiment dans l'expérience esthétique, faisant de celle-ci une sorte de pure épreuve affective. Mais, ce faisant, elles excluent aussi une motivation importante de la lecture, qui consiste à vivre, par les œuvres, ce que la vie actuelle n'offre pas - d'autres lieux ou d'autres temps, d'autres êtres et situations. Ingarden, en défendant la pureté de l'expérience esthétique contre tous les désirs qui, animant la lecture, pourraient venir la contaminer, discré-

\footnotetext{
${ }^{1}$ R. Ingarden, The Cognition of the Literary Work of Art, op. cit., § 32, p. 384 : «What is, or should be, the real goal (or end) of the aesthetic experience begins to be misused as a means to other, generally nonaesthetic, ends : a means of enriching our life, of being in certain states which are somehow pleasant for us. »

${ }^{2}$ Comme le remarque Menachem Brinker, dans son article «Roman Ingarden and the "Aesthetic Appropriate Attitude" to the Literary Work of Art », l'identification, par Ingarden, d'émotions extra-esthétiques, telles que les enjeux éthiques de la lecture sont posés comme étrangers à l'accomplissement de la lecture comme expérience esthétique, « is exaggerated. It is based on an aesthetic prejudice desire to model the reading experience on an aesthetic tailored to suit all of the arts and perhaps even natural objects » (Poetics Today, Vol. 5, No. 1, 1984, p. 147).

${ }^{3}$ R. Ingarden, The Cognition of the Literary Work of Art, op. cit., § 32, p. 384.

${ }^{4}$ Ibid., § 32, p. 385 : « Cheap, empty, and sensational works call for animated and varied extra-aesthetic feelings and thus are able to win over naive and uncultivated consumers. »
} 
dite les lecteurs qui, de fait, trouvent dans les œuvres les ressources pour nourrir une vie de fait appauvrie en expérience. Ingarden, en essayant de tracer une ligne nette entre émotions esthétiques et émotions non-esthétiques appauvrit en retour, de notre point de vue, l'expérience de lecture qu'il réduit à être l'expérience des quatre strates qu'il a identifiées comme appartenant à l'œuvre. En excluant les émotions qu'il nomme "secondaires » ${ }^{1}$ parce qu'elles parasitent l'expérience de lecture - pour autant qu'elles repoussent à l'arrière-plan les émotions esthétiques, voire les empêchent même de surgir -, Ingarden récuse comme incompétente une certaine sensibilité, proche de ce que Hume nomme délicatesse de la passion, pour défendre la fécondité d'une autre manière d'être, faite de "self-control, serenity, and concentration $»^{2}$, autant de dispositions aussi valorisées par Hume lorsqu'il expose ce qu'il appelle la délicatesse du goût.

\footnotetext{
${ }^{1}$ Ibid., § 32, p. 385 : «It is desired by many experiencing subjects and is often incorrectly thought to be the aesthetic experience itself. But it is not only completely foreign to the aesthetic experience but also disturbs the full and free development of that experience. In many cases it is precisely the abundance of the secondary feelings arising from the aesthetic experience which weakens its effectiveness. »

${ }^{2}$ Ibid., § 32, p. 385.
} 


\subsection{Délicatesse du goût et émotions esthétiques}

Telle qu'il la présente dans l'essai déjà cité, « De la délicatesse du goût et de la passion ", la délicatesse du goût est le propre de celui qui est sensible à la beauté et à la laideur, bref, à la qualité esthétique de ce qu'il rencontre ${ }^{1}$. Or, chez Hume, ce qui doit conduire à privilégier la délicatesse du goût sur celle de la passion, c'est tout d'abord le fait qu'elle ne soumet pas celui qui la possède à la contingence des circonstances et à l'instabilité émotionnelle, mais qu'elle le rend capable de rechercher avec discernement les œuvres qui le satisfont esthétiquement et donc d'enrichir sa vie de plaisirs esthétiques. C'est en cela que cette délicatesse va de pair avec une certaine sagesse philosophique par laquelle, loin de se jeter corps et âme dans les tourments de l'existence, il s'agit de trouver son bonheur dans le raffinement de l'expérience $^{2}$. Or, ce dernier dépend étroitement d'une discipline de l'attention, par laquelle il devient possible de considérer la manière dont les choses sont bien plus que de réagir par trop spontanément à leur existence.

De même, Ingarden, lorsqu'il peint le bon lecteur, insiste sur le fait que ce dernier, pour être à la recherche d'émotions esthétiques, demeure attentif, dans l'œuvre, à la forme, sans jamais se laisser obnubiler, sans distance, par ce qu'elle invite à figurer. Le propre de l'émotion esthétique, en effet, est de n'être pas motivée, exclusivement ou prioritairement, par ce qui est figuré dans l'œuvre littéraire mais par la manière dont cela est proposé à l'expérience. Ainsi, pour revenir à l'exemple précédemment donné - celui d'une expérience de contemplation picturale dans laquelle le spectateur est saisi par l'expression qui se dégage d'un portrait -, le spectateur ne revient à une attitude esthétique que si le fait d'être frappé par cette expression le renvoie, par exemple, à « un sentiment d'admiration pour la maîtrise du peintre » qui a réussi à rendre la personne figurée comme vivante ${ }^{3}$. Le spectateur passé dans l'attitude esthétique par ce retournement de l'attention, est donc celui qui se pose certaines questions devant l'œuvre - et non devant ce qu'elle met en scène. Citons, un peu longuement, Ingarden :

Le spectateur se demande comment il est possible que quelque chose de psychique comme un trait de caractère de la personne figurée devienne ainsi visible grâce à ces moyens-là ; et plus encore que quelque chose s'impose à

\footnotetext{
${ }^{1}$ D. Hume, « De la délicatesse du goût et de la passion », Essais sur l'art et le goût, op. cit., p. 67.

Ibid., p. 69.

${ }^{3}$ R. Ingarden, « De l'esthétique phénoménologique, essai de définition » (1969), art. cit., p. 59.
} 
lui avec une force telle qu'il n'est plus capable de se libérer de cette « impression », comme on dit parfois. Quels agencements de lignes et de couleurs sont-ils requis pour manifester le regard empreint d'amour et de bienveillance qu'une personne porte à une autre ? Le spectateur, qui se pose cette question et cherche la réponse en examinant plus avant le tableau, se transforme - et ici son comportement est essentiellement altéré —, d'un spectateur «naïf » qui échange simplement de manière émotionnelle avec les personnes figurées dans le tableau et qui réagit à leur comportement par son propre comportement de la même manière que dans les échanges personnels de la vie courante, il devient une personne qui considère la peinture donnée comme une auvre d'art, comme une formation singulière qui remplit des fonctions spéciales. Il procède maintenant à l'examen de ses couches spécifiques : ce qui est figuré et les moyens de la figuration, il examine de manière critique leurs fonctions et évalue leur efficience ou leur inefficacité artistique, finalement, soit il parvient à une haute évaluation de l'œuvre, soit il la rejette (la condamne) comme quelque chose de kitsch ${ }^{1}$.

Que la réaction émotionnelle née de la relation du spectateur à l'autre figuré cède la place à une émotion esthétique, cela signifie que le spectateur adopte une attitude dans laquelle, désormais, il ne considère plus ce qui est figuré mais le comment de la figuration et, plus que cela, une attitude dans laquelle celui qui est interrogé, ce n'est plus l'autre figuré mais l'artiste. En effet, en passant de l'attitude dite naïve à l'attitude esthétique, c'est aussi l'interlocuteur, pour le dire ainsi, du récepteur qui change, puisque ce dernier convoque, comme son vis-à-vis, celui qui a créé l'œuvre et dont il interroge la compétence, notamment technique. Autre façon de dire qu'une attitude esthétiquement compétente est aussi, pour Ingarden, une attitude qui pose la question de la compétence artistique et donc qui se tient sur le terrain de l'évaluation.

Surtout, Ingarden, en mettant en avant l'émotion esthétique, valorise le choc. Cela lui permet de décrire, ou de mettre en scène, le passage de l'expérience naïve à l'attitude esthétique et donc de poser, corrélativement, leur extériorité réciproque, leur étrangeté l'une à l'autre. La distinction entre émotions esthétiques et émotions extra-esthétiques recoupe la distinction entre attitude esthétique et attitude naïve ou de consommation, qui recoupe la distinction entre les lecteurs eux-mêmes, certains étant capables d'une relation désintéressée à l'œuvre tandis que d'autres continueraient, à même leur expérience de l'œuvre de faire valoir les intérêts qui animent leur vie

${ }^{1}$ Ibid., p. 60. 
pratique $^{1}$. Au contraire, le bon lecteur est celui qui est capable, à un moment, d'être saisi par une émotion provenant d'une certaine qualité présente dans l'œuvre. Cette émotion originale ( original emotion $»^{2}$ ) ou originelle en ce qu'elle ouvre la dimension esthétique de la lecture, provoque une rupture avec l'expérience actuelle :

A certain narrowing of the field of consciousness relating to this world takes place, although we do not lose the involuntarily feeling of its presence and existence, and we continue to feel that we are in the world. Nevertheless, our conviction of the world, which constantly colors our actuality, is shifted, to a certain extent, to the periphery of our consciousness or loses weight and force $^{3}$.

Le passage à l'attitude esthétique se joue comme mise hors-jeu de toute préoccupation pour l'existence du monde et des intérêts qu'il suscite. Or l'éloignement du monde actuel signifie aussi un retrait à l'arrière-plan des horizons de l'expérience présente, de telle sorte que le lecteur passé dans l'attitude esthétique perd le fil de sa vie propre ${ }^{4}$. C'est ainsi que le lecteur doit devenir capable d'être attentif à ce qui est étranger à sa vie personnelle tout autant que de laisser celle-ci sans poids sur la concrétisation effectuée. Autrement dit, l'imagination du bon lecteur, telle qu'elle est sollicitée par l'œuvre littéraire, ne saurait intervenir comme un plaquage de son imaginaire propre; bien au contraire, l'efflorescence des qualités esthétiques doit être motivée par son attention aux différentes strates de l'œuvre - notamment, répétons-le, à la strate des significations qui doit guider la constitution de la strate des objets et des aspects, donc les actes de présentification opérés par le lecteur. Parce que l'émotion esthétique est provoquée par ce que vit le lecteur en lisant, elle dépend, en effet, de la concrétisation qu'il effectue et donc de la manière dont il met en œuvre son imagination. Le lecteur qui ne sait pas imaginer correctement, pour le dire ainsi, est comme fermé par avance au surgissement de l'émotion esthétique, au passage de seuil qu'elle permet.

\footnotetext{
${ }^{1}$ R. Ingarden, The Cognition of the Literary Work of Art, op. cit., § 24, p. 188. Pour Ingarden, un passage doit bien s'opérer entre l'attitude naturelle dans laquelle se déroule la vie pratique et l'attitude esthétique, de telle sorte que les préoccupations de la vie pratique, si elles interviennent dans l'expérience qui est faite de l'œuvre d'art, ne peuvent être conçues dès lors que comme parasitaires.

${ }^{2}$ Ibid., $\$ 24$, p. 189.

${ }^{3}$ Ibid., $\S 24$, p. 192

${ }^{4}$ Ibid., § 24, p. 194
} 
Le lecteur qui a pu, ou qui a su, opérer le passage à l'attitude esthétique n'est pas en relation, comme l'est le consommateur de romans, avec une intrigue, mais avec un objet esthétique ${ }^{1}$ - celui-là même que son imagination, correctement employée, lui a permis de constituer ${ }^{2}$. Contrairement au lecteur naïf, prisonnier de son imaginaire et de ses intérêts, le bon lecteur n'est pas aux prises avec une intrigue, des personnages ou des situations humaines, mais bien alors avec des qualités esthétiques. Dans une certaine mesure, il a donc accès à quelque chose qui ne relève pas de ce dont l'œuvre parle ou de qu'elle invite à figurer, mais qui renvoie aux qualités purement esthétiques qui se dégagent de tout cela - clarté, subtilité, comique, tragique, banalité, profondeur, beauté, laideur, etc. ${ }^{3}$ Dans la mesure où ces qualités sont bien celles de l'œuvre dans la concrétisation effectuée par ce lecteur - selon les lignes, aussi, de sa sensibilité propre - , il est en contact avec des qualités qui, pour être attribuées à l'œuvre elle-même et être motivées par elle, ne peuvent émerger que sur le fondement des divers actes d'imagination qu'il a effectués, pour autant que ces actes d'imagination ne visaient pas à lui donner, par procuration, les plaisirs d'une vie imaginaire.

\subsection{Une imagination éduquée}

$\mathrm{Au} \S 28$ de The Cognition of the Literary Work of Art, Ingarden insiste bien sur le fait que l'expérience de lecture ne peut que provoquer des sentiments et des émotions qui ne relèvent pas directement d'une approche esthétique ${ }^{4}$ et qui sont susceptibles de faire obstacle à l'appréciation esthétique de

\footnotetext{
${ }^{1}$ Ibid., § 26, p. 224.

${ }^{2}$ R. Ingarden, «Valeurs esthétiques et valeurs artistiques », art. cit., p. 144 : « Si une concrétisation a lieu dans le cadre de l'attitude esthétique, alors apparait ce que j'appelle un objet esthétique. Cet objet ressemblera ou offrira le même caractère que ce qui était présent à l'esprit de l'artiste lorsqu'il a créé l'œuvre, si la concrétisation est effectuée avec l'intention de se conformer aux caractéristiques réelles de l'œuvre et de respecter les indications qu'elle donne autant que les limites du remplissement autorisé ». La bonne concrétisation donne lieu à la constitution d'un objet esthétique légitime, qui est approché ici comme analogue à celui que l'artiste devait avoir en vue au moment de la création. Le bon lecteur, ainsi, n'est pas seulement co-créateur de l'œuvre dans la seule mesure où il la concrétise mais, dans une certaine mesure, il égale en finesse esthétique l'artiste lui-même.

3 R. Ingarden, "La valeur esthétique et le problème de son fondement», in Esthétique et ontologie de l'œuvre d'art, choix de textes 1937-1969, op. cit., p. 135. ${ }^{4}$ R. Ingarden, The Cognition of the Literary Work of Art, op. cit., § 28, p. 306.
} 
l'œuvre d'art littéraire. Ce pourquoi Ingarden recommande d'éliminer (eliminate) ces émotions afin de préserver l'expérience esthétique ${ }^{1}$. La part qu'il y a ainsi en chacun de mauvais lecteur, affecté par les êtres et les situations qu'il découvre à la lecture d'une œuvre littéraire, doit être mise hors-jeu, au profit d'une attitude réflexive capable de faire le partage entre émotions naïves et émotions esthétiques, les premières risquant de contaminer la juste expérience et la juste évaluation de l'œuvre. Le bon lecteur est donc celui, avant tout, qui sait distinguer entre ses émotions afin de privilégier celles qui naissent de la structure, polystratique, de l'œuvre d'art littéraire elle-même et qui ne consistent pas exclusivement en réactions émotives à ce qu'il s'est figuré en augmentant ce dont l'œuvre parle de déterminations qui relèvent de l'arbitraire de son imagination. Autrement dit, le bon lecteur est capable d'une certaine distance eu égard à ses propres actes d'imagination, qui lui permet de questionner la pertinence même de sa performance et de distinguer entre ce qui, au sein même de son expérience de lecture, le touche personnellement et le touche esthétiquement - c'est-à-dire aussi devrait toucher tout autre, dans une juste appréhension de l'œuvre. Il est aussi celui, nous l'avons indiqué plus haut, qui «tient» sa lecture, en s'efforçant de tenir ensemble les diverses strates de l'œuvre et donc en contrôlant, au fur et à mesure de sa lecture, la cohérence et la fidélité des actes qu'il accomplit, notamment la consistance de ses actes présentifiants eu égard à la strate des significations.

Tout dépend donc de la manière dont le lecteur mène avec délicatesse sa lecture et, plus particulièrement, de la manière dont il accomplit les divers actes de présentification dans le but de faire émerger les qualités esthétiques de l'œuvre :

In other words, it depends on whether he has the aesthetic tact to admit only those ways of filling out places of indeterminacy which harmonize with one another (or possibly lead to desired contrasts) and which are not aesthetically dead but are active in the sense that they either contain aesthetically relevant qualities themselves or are the sufficient condition for constituting such qualities $^{2}$.

Or cette délicatesse ou ce tact ne se développent pas seulement avec la bonne intention de concrétiser l'œuvre adéquatement. Même si Ingarden affirme

${ }^{1} \mathrm{Ibid}, \S 28$, p. 306 .

${ }^{2}$ Ibid., $\S 32$, p. 373. 
que la lecture littéraire est un art et donc qu'elle s'apprend par l'exercice ${ }^{1}$, il revient en effet aussitôt sur cette affirmation pour dire qu'à l'art dont est capable le lecteur s'ajoute une certaine dose de «talent» qui ne s'apprend pas $^{2}$.

\section{Conclusion}

L'approche de l'expérience de lecture littéraire proposée par Ingarden articule donc ontologie de l'œuvre d'art, phénoménologie de la lecture mais aussi théorie du jugement de goût dans la mesure où son questionnement, in fine, vise à proposer les critères d'une juste expérience des œuvres et par conséquent d'une juste évaluation de leur valeur esthétique. Dans cette perspective, les développements qui concernent l'imagination, et les manières dont elle est mise à contribution dans l'expérience de lecture littéraire, possèdent aussi une dimension prescriptive, puisqu'il s'agit d'indiquer comment doit fonctionner l'imagination pour permettre de concrétiser avec pertinence et finesse l'œuvre littéraire. La position adoptée par Ingarden, en cela, n'est pas en rupture avec l'esthétique classique et, notamment, comme nous avons essayé de le développer ici, dans le prolongement des indications données par Patricia Limido-Heulot, de l'esthétique de Hume.

Cependant, contrairement à Hume, qui problématise la continuité qu'il peut y avoir entre finesse esthétique et finesse éthique, la délicatesse du goût étant un instrument d'appréciation des œuvres tout autant que des caractères - de telle sorte qu'elle doit aussi nous permettre de nous orienter dans nos relations avec les autres ${ }^{3}-$, Ingarden trace une frontière stricte entre émotions esthétiques et émotions extra-esthétiques et, pour cela, entre un usage esthétique de l'imagination, qui doit permettre de concrétiser l'œuvre pour faire ressortir ses qualités esthétiques, et un usage pathologique de l'imagination qui se prend au piège de l'intrigue et se laisse fasciner par les personnages. Par ce choix, qui tient à la volonté de faire de la relation du lecteur à l'œuvre une relation "de pure contemplation" ${ }^{4}$, et bien que les développements d'Ingarden concernant l'œuvre d'art littéraire et l'expérience

\footnotetext{
${ }^{1} \mathrm{Ibid}, \S 28$, p. 309. Cela, notamment, en pratiquant la relecture par laquelle il est possible de s'entraîner à mieux comprendre et, par suite, à présentifier avec plus de pertinence.

${ }^{2}$ Idem.

${ }^{3}$ D. Hume, « De la délicatesse du goût et de la passion », Essais sur l'art et le goût, op. cit., p. 69.

${ }^{4}$ R. Ingarden, L'Cuvre d'art littéraire, op. cit., p. 284.
} 
de lecture soient féconds, ils sont aveugles à certaines dimensions de l'expérience de lecture, qui font la richesse de la lecture et la valeur de la littérature pour ses lecteurs, et qui concernent la manière dont cette dernière enrichit la vie éthique de ces derniers.

Par ailleurs, Ingarden est moins précis que Hume dans l'exposition des conditions empiriques qui doivent permettre au lecteur de développer une imagination délicate, susceptible d'être sensible à la valeur esthétique des œuvres rencontrées, voire il est moins rigoureux sur la reconnaissance de l'importance de ces conditions. S'il insiste sur la nécessité de reconnaître un partage entre ceux qui savent apprécier les œuvres littéraires et ceux qui ne savent pas - de telle sorte que ces derniers ne doivent pas avoir droit au chapitre quand on en vient à l'évaluation des œuvres d'art littéraires ${ }^{1}-$, il ne dessine pas fermement ce en quoi doit consister une éducation esthétique. Plus que cela, il semble hésiter à affirmer la nécessité d'une éducation esthétique et à reconnaître le poids, pour ce faire, des institutions pour laisser la place à la figure de l'amateur.

The sensitive, effective, and faithful aesthetic experience never seeks out some privileged circles of the community; in principle, it is accessible to everyone, in the sense that it can be awakened and even, to a certain extent, taught and exercised in contact with great works of art ${ }^{2}$.

Ingarden reconnait certes qu'on peut apprendre à apprécier les œuvres littéraires, voire qu'il est possible de s'exercer à les concrétiser et à les apprécier, mais il semble pourtant privilégier, par contraste avec cela, l'événement du passage à l'attitude esthétique voire l'événement du passage à la délicatesse pour celui qui aurait été touché par une sorte de grâce. Ce privilège accordé à l'événement esthétique lui permet, d'une part, de sauvegarder la pertinence de l'approche phénoménologique, dans la mesure où celle-ci n'a pas à être articulée à une histoire de la lecture et à une didactique de la lecture : sa phénoménologie peut ainsi se déployer comme la description de l'expérience de certains, et se justifier en affirmant que cette expérience est par principe accessible à tous, parce que l'accès à cette expérience ne dépendrait pas fermement de certaines conditions empiriques mais de l'événement, imprévisible, d'une rencontre entre l'œuvre et le lecteur. C'est ainsi que ce privilège accordé à l'événement lui permet, aussi, d'ignorer les enjeux politiques de l'accès à l'art et de l'éducation de la sensibilité et de l'imagi-

\footnotetext{
${ }^{1}$ R. Ingarden, The Cognition of the Literary Work of Art, op. cit., § 33, p. 418.

${ }^{2}$ Idem.
} 
nation. En faisant de l'accès à l'attitude esthétique l'enjeu d'une «révélation ${ }^{1}$, l'approche d'Ingarden légitime les inégalités de fait entre les récepteurs et affirme l'impossible partage des expériences de lectures. Les œuvres d'art littéraires séparent les lecteurs: ceux-ci lisent depuis des attitudes étrangères les unes aux autres, jouent différemment de leur imagination, éprouvent, par elle, des émotions qui ne sauraient légitimement être mises en relation et n'ont, finalement, rien à se dire.

\section{Bibliographie}

Brinker M., «Roman Ingarden and the "Aesthetic Appropriate Attitude" to the Literary Work af Art », Poetics Today, Vol. 5, No. 1, 1984, p. 129-148.

Clark Walter H., "Review of The Cognition of the Literary Work of Art », The Journal of Aesthetics and Art Criticism, Vol. 33, No. 2, Winter, 1974, p. 220222.

Hume D., « De la règle du goût », Essais sur l'art et le goût, tr. fr. M. Malherbe, Paris, Vrin, 2010.

Husserl E., Recherches logiques II, t. 2, tr. fr. H. Elie, A. Kelkel et R. Schérer, Paris, P.U.F, 1969

Ingarden R., Esthétique et ontologie de l'œuvre d'art : Choix de textes 1937-1969, tr. fr. P. Limido-Heulot, Paris, Vrin, 2011.

Ingarden R., L'Euvre d'art littéraire, tr. fr. P. Secretan avec la collaboration de N. Lüchinger et B. Schwegler, Lausanne, L'Âge d'Homme, 1983.

Ingarden R., The Cognition of the Literary Work of Art, tr. fr. R. A. Crowley et K.R. Olson, Evanston, Northwestern University Press, 1973.

Limido-Heulot P., «L'esthétique phénoménologique de Roman Ingarden», introduction à Esthétique et ontologie de l'œuvre d'art: choix de textes 19371969, Paris, Vrin, 2011, p. 7-23.

Merleau-Ponty M., La Prose du monde, Paris, Gallimard, 1969.

${ }^{1}$ « But just as not everyone has to be a mathematician, so not everyone has to have an understanding of art, although it is regrettable when the revelation of art and of the value concretized thereby is not successful in a particular case » (Idem). 